\title{
Recommendations for the clinical management of children with refractory epilepsy receiving the ketogenic diet
}

\author{
María J. Alberti, M.D. ${ }^{a}$, ArielaAgustinho, M.D. ${ }^{b}$, Laura Argumedo, M.D. ${ }^{c}$, \\ Marisa Armeno, M.D. ${ }^{b}$, Virginia Blanco, B.S. ${ }^{d}$, Cecilia Bouquet, M.D. ${ }^{e}$, \\ Analía Cabrera, M.D. ${ }^{d}$, Roberto Caraballo, M.D. ${ }^{b}$, Luciana Caramuta, M.D. ${ }^{e}$, \\ Araceli Cresta, B.S. ${ }^{b}$, Elizabeth S. de Grandis, M.D. ${ }^{f}$, Martha G. De Martini, M.D. ${ }^{g}$, \\ Cecilia Diez, M.D. ${ }^{h}$, Corina Dlugoszewski, M.D. ${ }^{i}$, Nidia Escobal, M.D. ${ }^{b}$, \\ Hilario Ferrero, M.D. ${ }^{j}$, Santiago Galicchio, M.D. ${ }^{d}$, Victoria Gambarini, B.S. ${ }^{i}$, \\ Beatriz Gamboni, M.D. ', Silvina Guisande, B.S. ${ }^{e}$, Amal Hassan, M.D. ${ }^{c}$, Pablo Matarrese, M.D. ${ }^{a}$, \\ Graciela Mestre, B.S. ${ }^{b}$, Laura Pesce, B.S. ', Viviana Ríos, M.D. ${ }^{g}$, Patricia Sosa, M.D. ${ }^{e}$, \\ María Vaccarezza, M.D. ${ }^{h}$, RocíoViollaz, B.S. ${ }^{a}$, and Luis Panico, M.D. ${ }^{(+)}$
}

a. Hospital de Niños Sor María Ludovica, La Plata, Buenos Aires.

b. Hospital Nacional de Pediatría "Dr. Prof. Juan P. Garrahan", Buenos Aires.

c Hospital Pediátrico Doctor Humberto J. Notti, Mendoza.

d. Hospital de Niños Víctor J. Villela, Rosario.

e. Hospital Nacional Profesor Alejandro Posadas, Buenos Aires.

f. Hospital de Niños de la Santísima Trinidad, Córdoba.

g. Hospital de Niños Doctor Orlando Alassia, Santa Fe.

h. Hospital Italiano de Buenos Aires.

i. Hospital de Niños Doctor Ricardo Gutiérrez, Buenos Aires.

j. Hospital Privado de Comunidad, Mar del Plata, Buenos Aires.

E-mail address:

María J. Alberti, M.D.: mjalberti@hotmail.com

Funding:

None.

Conflict of interest: None.

Received: 5-15-2015 Accepted: 9-18-2015

\section{ABSTRACT}

The ketogenic diet, a non-drug treatment with proven effectiveness, has been the most commonly used therapy in the past decade for the managementof refractory epilepsy in the pediatric population. Compared to adding a new drug to a pre-existing treatment, the ketogenic diet is highly effective and reduces the number of seizures by $50-90 \%$ in approximately $45-60 \%$ of children after six months of treatment. For this reason, the Argentine Society of Pediatric Neurology established the Ketogenic Diet Working Group. It is integrated by pediatric dietitians, pediatricians, pediatric neurologists and B.S. in Nutrition, who developed recommendations for the optimal management of patients receiving the classical ketogenic diet based on expert consensus and scientific publications in this field.

Key words: ketogenic diet, refractory epilepsy, pediatrics.

http://dx.doi.org/10.5546/aap.2016.eng.56

\section{INTRODUCTION}

Epilepsy is a disorder that occurs in $0.5-1 \%$ of the world population, and the onset of $60 \%$ of epilepsy cases occurs during childhood. ${ }^{1}$

The prevalence of refractory epilepsy (defined as seizures that cannot be managed with at least two first line antiepileptic drugs in adequate doses, as single or combined drug therapy) is still high, although multiple antiepileptic agents have been developed over the past 10 years. Epidemiological data indicate that $20-30 \%$ of patients with epilepsy will become refractory to treatment. ${ }^{2}$ There are many factors that may result in failure of conventional therapies, such as the different types and etiologies of epilepsy.

Drug-resistance is therefore a multi-cause phenomenon, and the use of alternative therapies may be an adequate choice to improve results in this population of patients.

The classical ketogenic diet is a high-fat, adequate-protein, lowcarbohydrate diet designed to cause ketosis and to mimic metabolic changes due to fasting. ${ }^{2,3}$

In relation to its action, it is now increasingly evident that the ketogenic diet works throughmultiple mechanisms that target fundamental biochemical pathways linked to cell substrates (e.g., ion channels) and mediators responsible for neuronal hyperexcitability.

Many hypotheses have been proposed, including the direct anticonvulsant effect of ketone bodies, changes in neurotransmitter, gammaaminobutyric acid (GABA), glutamate and adenosine systems, as well as ion channel regulation, improved cellular bioenergetics and mitochondrial function, inhibitory actions of fatty acids, reduced oxidative stress, and improved tricarboxylic acid function using dietary supplementation with anaplerotic substrate. ${ }^{4}$

Fasting as a method to achieve seizure control was described approximately 90 years ago. ${ }^{5} \mathrm{Next}$, with the emergence of multiple drugs, this diet fell into disuse; more recently, 
in the 1990s, it has re-emerged together with vagus nerve stimulation and epilepsy surgery as a potential response to epilepsy patients who did not respond to isolated drug therapy. Thus, at present, the ketogenic diet (in its classical, medium-chain triglyceride, modified Atkins, and low-glycemic index modalities) plays a relevant role in the treatment of these patients, and it is being with proven effectiveness in approximately 200 sites worldwide. ${ }^{2,3}$ Treatment with the classical ketogenic diet has shown a close to $50 \%$ reduction in the number of seizures in half of patients, and a $90 \%$ seizure reduction in one third of patients. ${ }^{2,3,6}$

The ketogenic diet, a non-drug treatment with proven effectiveness, has been the most commonly used therapy in the past decade for the management of refractory epilepsy in the pediatric population. This poses a new challenge to pediatricians, neurologists, general practitioners and therapists, who now more and more have to see patients who are on this treatment and who, like any other child, may get sick, require medications and procedures that imply fasting and/or anesthesia, including surgeries.

Like any other antiepileptic treatment, it is not exempt from complications, which should be known, monitored and prevented through an adequate follow-up and a timely intervention.

In Argentina, the ketogenic diet has been used for two decades in an increasing number of sites. In 2011, the first multicenter study, conducted in 216 patients, was published. ${ }^{12}$ In 2012, the Argentine Society of Pediatric Neurology (Sociedad Argentina de Neurología Infantil) established the Ketogenic Diet Working Group. It is made up of pediatric dietitians, pediatricians, pediatric neurologists and sites located in five Argentine provinces. In 2014, the group published the first national consensus on the ketogenic diet to standardize its use at the different sites. ${ }^{1}$

At present, a national registry of patients receiving treatment is underway.

\section{Indications and contraindications}

The ketogenic diet is prescribed by a pediatric neurologist, who is in charge of establishing which patients may be candidates for this treatment. ${ }^{2,6,7}$ This indication is completed with a series of interviews (pre-admission) by the team, who provide an interdisciplinary comprehensive assessment of the patient and his/her adverse limiting or risk factors to duly adhere to the ketogenic diet. ${ }^{1}$ For patients with a glucose transporter protein type 1 (GLUT1) deficiency or a pyruvate dehydrogenase (PDH) deficiency, the classical ketogenic diet is the treatment of choice. ${ }^{7}$ In the case of refractory epilepsy, results vary and are better with certain types of seizures or epileptic syndromes (Table 1). Patient selection and assessment are essential to achieve the best clinical management possible and reach the desired objective.

It is also necessary to rule out clinical conditions for which the ketogenic diet is contraindicated (Table 2) and assess risk factors that may complicate the diet once it has been started (e.g., gastroesophageal reflux). ${ }^{1,3,8}$

\section{Preparation for the diet, medication and supplementation}

Once it has been established that the patient has an indication for the ketogenic diet, it is necessary to schedule an initial appointment with the patient and his/her caregivers for an assessment by the multidisciplinary team, which is made up of a pediatric neurologist, a dietitian and a B.S. in Nutrition. ${ }^{1}$

TABLE 1. Indications for the ketogenic diet

- Failure to control seizures after adding a second medication.

- Medically refractory epilepsy.

- First-line treatment for:

- GLUT1 deficiency.

- PDH deficiency.

- Metabolic disorders:

- Phosphofructokinase deficiency.

- Glycogen storage disease type V.

- Mitochondrial respiratory chain complex disorders.

- Epileptic syndromes:

- Myoclonic astatic epilepsy.

- Seizures caused by tuberous sclerosis complex.

- West syndrome refractory to vigabatrin or adrenocorticotropic hormone (ACTH).

- Dravet syndrome.

- Symptomatic epilepsies:

- Lafora body disease.

- Seizures caused by Rett syndrome.

- Landau-Kleffner syndrome.

- Sub acute sclerosing panencephalitis.

- Febrile infection-related epilepsy syndrome (FIRES).

- Refractory status epilepticus. 
It is critical to establish that the patient (if older enough) and his/her family are able to understand the treatment, given that a strict adherence with no transgressions ensures effectiveness and the possibility to remain under a long-term treatment. ${ }^{8}$

The pediatric neurologist makes a detailed examination and assesses seizure characteristics (type, duration, daily average) and the type and dose of medication the patient is receiving. $\mathrm{He} / \mathrm{she}$ is also responsible for prescribing lowcarbohydrate formulations. It is also important to record all previous studies and establish the type of epilepsy or epileptic syndrome.

During the nutritional assessment, the patient's personal or family history of kidney stones or dyslipemia is evaluated. Once a detailed case history and a three-day diet record are available, meal preferences, allergies, dislikes and intolerance are explored. During the patient's physical exam, an assessment of nutritional status is done and anthropometric measures are collected. It is very important to assess swallowing so as to establish a safe feeding pathway (oral, nasogastric tube or gastrostomy) and decide on the diet formulation to be used (formula or food for an adequate recipe preparation).

Fluid, calorie and protein intake is then estimated based on the patient's age, nutritional status, activity level and preferences. Lastly, the type of diet and the ketogenic ratio are established. ${ }^{9}$

In general, the diet starts with a 4:1 ratio, i.e., $90 \%$ of energy coming from fat and $10 \%$, from protein plus carbohydrate.

In young or obese children or those who have an excessive ketosis production during implementation, a 3:1 ratio may be used. In this

\section{TABLE 2. Contraindications for the ketogenic diet}

\section{- Absolute}

- Disorders of fatty acid transport.

- Disorders of fatty acid oxidation (beta-oxidation).

- Pyruvate carboxylase deficiency.

- Porphyria

- Moderate or severe malnutrition.

- Relative:

- Unstable familial psychological structure.

- Precarious social stratum.

- Epilepsy with a clear surgical focus as established by neuroimaging and video-electroencephalogram monitoring. case, $87 \%$ of energy comes from fat and $13 \%$, from protein plus carbohydrate.

Before starting the diet, lab tests are requested (Table 3) to assess the patient's metabolic status and any prior alteration. ${ }^{1,2}$

The classical ketogenic diet lacks in minerals and vitamins ${ }^{3,10}$ due to the little variability in macronutrient offered by this treatment; therefore, patients on this diet should always use supplements with carbohydrate-free formulations (Table 4). It is especially important to administer calcium supplements because these children have a risk of developing osteopenia and osteoporosis due to the long-term use of anticonvulsant agents and metabolic acidosis with compensatory hypercalciuria generated by the diet. ${ }^{11,12}$ Exposure to the sun should also be encouraged, accompanied by vitamin D supplementation, if necessary. It is important to routinely monitor bone mineral density and phosphocalcic metabolism.

\section{Initiation}

a) With hospitalization or as outpatient:

Studies have not found significant differences between these two modalities in terms of seizure control; $; 13,14$ however, avoiding a hospitalization without having to fast may reduce the child's stress. In addition, the risk of hypoglycemia and dehydration, the number of lab tests and costs are reduced with the outpatient modality. If the family is trained as per this modality, it is necessary to have an outpatient clinic or metabolic unit where the patient's blood glucose and urine ketones can be controlled, and families receive training on how to prepare the diet and manage techniques required by the treatment, and it is possible to monitor for adverse effects, if any.

TABLE 3. Supplements used during the ketogenic diet

\footnotetext{
Routine:

- Multivitamins, minerals and trace elements.

- Calcium and vitamin D.

- Iron.
}

Optional, depending on individual circumstances:

- Potassium citrate, with 24-hour urine calcium $4 \mathrm{mg} / \mathrm{kg}$ $(0.1 \mathrm{mmol} / \mathrm{kg})$ or calcium $/$ creatinine in isolated sample $0.2 \mathrm{mg} / \mathrm{mg}$ or $0.6 \mathrm{mmol} / \mathrm{mmol}$.

- Laxatives.

- Medium-chain triglycerides (MCT).

- Selenium, magnesium, zinc, phosphorus, additional bicarbonate.

- Carnitine (with moderate deficit or long-term treatment with valproic acid). 
b) Hospitalization schedule (if necessary):

During the initiation of the diet, intake may be increased according to the experience of the site's staff, with full calories early on, progressing from a 2:1 ratio, or starting with a 4:1 ratio and progressively increasing calories.

Initial fasting (with no carbohydrate consumption) is not routinely recommended; it should only be considered for specific situations, e.g., for critically-ill patients. Although ketosis may be induced after a short fasting period, it is not strictly necessary to cause and maintain a metabolic condition of ketonemia. The benefit of fasting is mostly related to shortening the time necessary to achieve ketosis, but its disadvantages include a risk of hypoglycemia and dehydration, psychological stress, increased hospitalization costs and more blood sample collections. . $^{15,16}$

c) Medical controls during hospitalization

Blood glucose and urine ketones are checked every 8 hours in the first 48 hours of diet

\section{TABLE 4. Lab tests and additional procedures}

- Complete blood count.

- Blood electrolytes.

- Serum bicarbonate (acid-base status).

- Total protein, albumin.

- Blood glucose.

- Calcium, magnesium and phosphorus.

- Fasting lipid profile.

- Zinc and selenium (as available).

- Renal function (blood urea and creatinine).

- Liver function (glutamic oxalacetic transaminase [GOT], glutamic pyruvic transaminase [GPT], prothrombin).

- Urinalysis with calcium and creatinine levels (Ca/Cr ratio).

Specific tests:

- Anticonvulsant blood levels (if necessary).

- Urine organic acids.

- Serum acylcarnitine profile.

- Lactic acid, pyruvic acid, and beta-hydroxybutyrate, fasting and postprandial.

- Ancillary tests (optional):

- Renal ultrasonography, visit to the nephrology department (in case of history of kidney stones or treatment with topiramate).

- Abdominal ultrasonography.

- Wrist X-ray (bone age).

- Electrocardiogram with QTc. Echocardiogram.

- Electroencephalogram.

- Magnetic resonance imaging (MRI).

- Cerebrospinal fluid (in case of uncertain etiology). initiation. If signs or symptoms of hypoglycemia are observed, e.g., agitation, cold sweats, pallor, disturbance of consciousness or sleeping disorders, controls should be more frequent. In the case of asymptomatic blood glucose below $0.4 \mathrm{~g} / \mathrm{L}, 10-20$ $\mathrm{mL}$ of orange juice should be administeredorally. If symptomatic, a $5 \%$ dextrose infusion at $4 \mathrm{~mL} /$ $\mathrm{kg} /$ hour should be administered.

In the case of persistent hypoglycemia associated with a delay in achieving ketosis, a congenital metabolic error should be suspected. This is particularly the case with enzymatic disorders of fatty acid beta-oxidation.

d) Diet adjustments

- Progressive increase of ketogenic ratio.

- Assessment and changes due to the patient's preferences or intolerance.

e) Family training:

- Education on diet concepts.

- Use of the gram scale.

- Recipe preparation.

- Food storage techniques.

- Training on how to measure urine ketone bodies.

- Preparation of a ketosis/seizure sheet.

- Delivery of the form to be submitted at the E.R. or before a hospitalization (Annex).

\section{Short- and long-term adverse effects}

The ketogenic diet is not a harmless treatment. ${ }^{17-19}$ It is a meal plan with an unbalanced intake of micro- and macronutrients; it may result in energy, protein, mineral and vitamin deficiency and excessive lipid intake, with a risk of untoward side effects. However, its effects are not usually severe, and few patients need to interrupt the diet.

During the implementation phase of the ketogenic diet, the more common complications are metabolic acidosis and gastrointestinal manifestations, such as abdominal pain, nausea and vomiting with a risk of dehydration and hypoglycemia, ${ }^{17-19}$ especially in patients who remain fasting for an extended period of time. Less common, but very important, effects because of their difficult management include eating disorders, such as loss of appetite, solid food and/or fluid rejection, and self-induced vomiting.

During the maintenance phase, common complications observed are dyslipemia and gastrointestinal alterations, including constipation, nausea and exacerbation of the 
gastroesophageal reflux. ${ }^{20,21}$ During this phase, metabolic alterations may also occur, such as acidosis, hypokalemia, hypomagnesemia, carnitine deficiency (especially in patients receiving valproic acid), anemia, and trace element deficiency, including selenium, copper and others. ${ }^{10,18}$ All these manifestations should be prevented through strict monitoring and an adequate micronutrient supplementation.

A special consideration should be given to bone alterations, since patients on the ketogenic diet are a population with a high risk of developing osteopenia. This is because anticonvulsant agents interfere with the phosphocalcic and vitamin D metabolism. In addition, many of these patients are not able to walk or be exposed to the sunlight and develop acidosis. ${ }^{11,12}$

Kidney stones, pancreatitis and gallstones are less common. It is worth noting that it is difficult to establish whether those complications are related to the ketogenic diet or anticonvulsant agents, or are secondary to the underlying disease. 22,23

QT prolongation is generally associated with selenium deficit and metabolic acidosis. Although common, it is severe, so a regular heart examination is critical. ${ }^{24-26}$

In relation to the effect of the ketogenic diet on growth, the most recently published reports indicate that an adequate calorie and protein intake result in a normal growth rate. ${ }^{27,28}$ During each visit, anthropometric data are assessed and compared to growth patterns recommended by the Argentine Society of Pediatrics. ${ }^{29}$

Long-term complications have not yet been systematically reviewed.

\section{Post-hospitalization medical controls}

A joint nutritional and neurological control is recommended on the first week, and 15 days, and 1 month after treatment initiation, and then every 3 months (Table 5); however, in general, a close communication via e-mail or telephone is required throughout treatment.

Diet adherence is reviewed based on visit attendance and submission of a sheet where solid food, fluids, urine ketones and seizure frequency are recorded.

\section{Concurrent conditions (E.R. Department and surgeries)}

Infectious concurrent diseases are common during childhood, so it is not uncommon that children on the ketogenic diet seek care at the
E.R. Department, require an intervention or hospitalization.

After starting the diet, the treating team should give family members an explanatory form to be submitted to the staff should they take the patient to an E.R. Department or seek care in a different medical facility (Annex).

During the course of a disease, an unlimited quantity of fluid and no carbohydrate intake are recommended, as tolerated, in order to prevent dehydration caused by high fever or diarrhea. For prolonged vomiting (24-48 hours), oral rehydration salts and intravenous hydration may be used, but estimating carbohydrate and electrolyte amounts individually for each patient so as to prevent excessive ketosis and hypoglycemia.

Once the concurrent condition has resolved, the patient's usual ketogenic feeding may be gradually reintroduced, similarly to the diet initiation phase (half or a third of calories on the first day, with progressive increases over 2-3 days).

Patients who undergo brief surgeries should receive glucose-free intravenous hydration under strict surveillance. ${ }^{30}$ For extended surgical procedures (longer than 3 hours), blood glucose, electrolytes and blood $\mathrm{pH}$ should be monitored based on the risk of metabolic acidosis. It has been reported that glucose levels remain stable, while $\mathrm{pH}$ goes down, requiring correction of acidosis by sodium bicarbonate. The ketogenic diet should be started again as soon as possible.

If children require medications, these should be administered in carbohydrate-free formulations or formulations with the lowest carbohydrate amount possible. Since multiple formulations are available in the market, it is fundamental to ask the manufacturing pharmaceutical company about the ingredients in each medication that may be administered.

It has also been reported that oral glucocorticoids cause reduced ketosis, so they should be administered cautiously, only when the clinical picture requires so and in the lowest dose possible. In case of an emergency, such as a severe respiratory exacerbation or an allergic reaction, the use of high doses of steroids may require a transient interruption of the ketogenic diet, prioritizing the patient's life.

\section{Discontinuation}

The time and manner of discontinuation should be customized to each patient. ${ }^{31}$ 
If the diet is not effective and no adverse effects are observed, it should be discontinued after 6 months of treatment in the course of one week.

If treatment is effective, reduction should be started 2-3 years after initiation by decreasing 1 point in the ketogenic ratio every $2-3$ weeks. It is recommended to extend it for a longer period if seizure reduction with the diet is over $90 \%$, if the number of seizures increases during reduction

\section{TABle 5. Post-hospitalization controls}

\section{a. Nutritional control:}

- Weight, height, body mass index, head circumference, growth rate.

- Review of adherence to diet prescription (calories, proteins, fluids).

- Diet modification due to changes in weight, liking, intolerance.

- Review of supplementation (as per the reference daily intake [RDI]).

- Treatment adjustments to optimize adherence or seizure control.

- Assessment of favorite meals or food that may be incorporated.

- Control of meal times.

- Presence of anorexia.

- Bowel movements.

- Urinary ketosis controls.

\section{b. Neurological control:}

- Present average number of seizures and estimation of percentage decrease.

- Seizure type and duration. Anticonvulsant response as per Huttenlocher's modified criteria.

- Electroencephalogram result.

- Diet effectiveness (meets parents' expectations).

- Assessment of quality of life.

- Assessment of possible reduction in anticonvulsant agents.

c. Lab tests and additional procedures (depending on treatment timing):

- Quarterly control: blood count, total protein, blood glucose, blood creatinine, acid-base status, ionogram, urine, $\mathrm{Ca} / \mathrm{Cr}$ ratio.

- Six monthly control: in addition to the above mentioned controls, cholesterol, triglycerides, high-density lipoprotein (HDL), low-density lipoprotein (LDL), blood urea, blood uric acid, GPT, alkaline phosphatase, blood phosphorus, total and free carnitine (if available at the lab). Electrocardiogram with QTc and electroencephalogram.

- Annual control: in addition to the above mentioned controls, renal ultrasonography and wrist $\mathrm{X}$-ray. Bone densitometry. of the ketogenic ratio, or in patients with certain syndromes, such as tuberous sclerosis complex or Dravet syndrome.

\section{CONCLUSIONS}

The ketogenic diet is a safe and effective interdisciplinary approach to different types of epilepsy, but it should be implemented after a careful selection and education of patients and their families so as to favor a better treatment response and prevent adverse effects.

During the ketogenic diet, concurrent conditions or other diseases may occur that require a medical visit. Pediatricians are required to incorporate and offer an adequate management to this group of patients.

\section{REFERENCES}

1. Armeno M, Caraballo R, Vaccarezza M, Alberti MJ, et al. Consenso nacional sobre dieta cetogénica. Rev Neurol 2014;59(5):213-23.

2. Freeman JM, Kossoff EH, Hartman AL. The ketogenic diet: one decade later. Pediatrics 2007;119(3):535-43.

3. Neal EG, Chaffe H, Schwartz RH, Lawson MS, et al. The ketogenic diet for the treatment of childhood epilepsy: a randomised controlled trial. Lancet Neurol 2008;7(6):500-6.

4. Rho JM. How does the ketogenic diet induce anti-seizure effects? NeurosciLett 2015. Epub Jul 26.

5. Wheless JW. History of the ketogenic diet. Epilepsia2008;49(Suppl 8):3-5.

6. Lee PR, Kossoff EH. Dietary treatments for epilepsy: management guidelines for the general practitioner. Epilepsy Behav 2011;21(2):115-21.

7. Nangia S, Caraballo RH, Kang HC, Nordli D, et al. Is the ketogenic diet effective in specific epilepsy syndromes? Epilepsy Res 2012;100(3):252-7.

8. Kossoff EH,Zupec-Kania BA, Amark PE, Ballaban-Gil KR, et al. Optimal clinical management of children receiving the ketogenic diet: recommendations of the International Ketogenic Diet Study Group. Epilepsia 2009;50(2):304-17.

9. Seo JH, Lee YM, Lee JS, Kang HC, et al. Efficacy and tolerability of the ketogenic diet according to lipid: nonlipid ratios. Comparison of 3:1 with 4:1 diet. Epilepsia 2007;48(4):801-5.

10. Christodoulides SS, Neal EG, Fitzsimmons G, Chaffe H, et al. The effect of the classical and medium chain triglyceride ketogenic diet on vitamin and mineral levels. J Hum Nutr Diet 2012;25(1):16-26.

11. Bergqvist AG, Schall JI, Stallings VA, Zemel BS. Progressive bone mineral content loss in children with intractable epilepsy treated with the ketogenic diet. Am J Clin Nutr 2008;88(6):1678-84.

12. Caraballo RH, Vaccarezza M, Cerósimo R, Ríos V, et al. Long-term follow-up of the ketogenic diet for refractory epilepsy: multicenter Argentinean experience in 216 pediatric patients. Seizure 2011;20(8):640-5.

13. Rizzutti S, Ramos AM, Muszkat M, Gabbai AA. Is hospitalization really necessary during the introduction of the ketogenic diet? J Child Neurol 2007;22(1):33-7.

14. Vaisleib II, Buchhalter JR, Zupanc ML. Ketogenic diet: outpatient initiation, without fluid, or caloric restrictions. Pediatr Neurol 2004;31(3):198-202.

15. Bergqvist AG, Schall JI, Gallagher PR, Cnaan A, et al. Fasting versus gradual initiation of the ketogenic diet: a 
prospective, randomized clinical trial of efficacy. Epilepsia 2005;46(11):1810-9.

16. Kim DW, Kang HC, Park JC, Kim HD. Benefits of the non fastingketogenic diet compared with the initial fasting ketogenic diet. Pediatrics 2004;114(6):1627-30.

17. Kang HC, Chung DE, Kim DW, Kim HD. Early- and lateonset complications of the ketogenic diet for intractable epilepsy. Epilepsia 2004;45(9):1116-23.

18. Vining EP. Long-term health consequences of epilepsy diet treatments. Epilepsia 2008;49(Suppl 8):27-2.

19. Ballaban-Gil K, Callahan C, O'Dell C, Pappo M, et al. Complications of the ketogenic diet. Epilepsia 1998;39(7): 744-8.

20. Zupec-Kania B, Zupanc ML. Long-term management of the ketogenic diet: seizure monitoring, nutrition and supplementation. Epilepsia 2008;49(Suppl 8):23-6.

21. Groesbeck DK, Bluml RM, Kossoff EH. Long-term use of the ketogenic diet in the treatment of epilepsy. Dev Med Child Neurol 2006;48(12):978-81.

22. McNally MA, Pyzik PL, Rubenstein JE, Hamdy RF, et al. Empiric use of potassium citrate reduces kidneystone incidence with the ketogenic diet. Pediatrics 2009;124(2):e300-4.

23. Hartman AL, Vining EP. Clinical aspects of the ketogenic diet. Epilepsia 2007;48(1):31-42.
24. Best TH, Franz DN, Gilbert DL, Nelson DP, et al. Cardiac complications in pediatric patients on the ketogenic diet. Neurology 2000;54(12):2328-30.

25. Sharma S, GulatiS. The ketogenic diet and the QT interval. J Clin Neurosci 2012;19(1):181-2.

26. Sirikonda NS, Patten WD, Phillips JR, MullettCJ. Ketogenic diet: rapid onset of selenium deficiency-induced cardiac decompensation. Pediatr Cardiol 2012;33(5):834-8.

27. Peterson SJ, Tangney CC, Pimentel-Zablah EM, Hjelmgren $\mathrm{B}$, etal. Changes in growth and seizure reduction in children on the ketogenic diet as a treatment for intractable epilepsy. J Am Diet Assoc 2005;105(5):718-25.

28. Tagliabue A, Bertoli S, Trentani C, Borrelli P, et al. Effects of the ketogenic diet on nutritional status, resting energy expenditure, and substrate oxidation in patients with medically refractory epilepsy: a 6-month prospective observational study. ClinNutr 2012;31(2):246-9.

29. Sociedad Argentina de Pediatría. Comité Nacional de Crecimiento y Desarrollo. Guía para la evaluación del crecimiento físico. 3ra ed. Buenos Aires: Sociedad Argentina de Pediatría; 2013.

30. Valencia I, Pfeifer H, Thiele EA. General anesthesia and ketogenic diet: clinical experience in nine patients. Epilepsia 2002;43(5):525-9.

31. Worden LT, Turner Z, Pyzik PL, Rubenstein J, et al. Is there an ideal way to discontinue the ketogenic diet? Epilepsy Res 2011;95(3):232-6. 


\section{Annex}

\section{FORM TO BE SUBMITTED AT THE E.R. DEPARTMENT OR IF HOSPITALIZED IN A DIFFERENT FACILITY}

\section{Patient receiving the ketogenic diet}

\section{Treatment precautions during an emergency}

The ketogenic diet is a treatment for drug-refractory epilepsy that provides $90 \%$ of energy in the form of fat, with a protein level adequate for the patient's age and very little carbohydrates.

\section{THIS DIET SHOULD NOT BE ABRUPTLY INTERRUPTED.}

In the case of asymptomatic blood glucose levels below $0.4 \mathrm{~g} / \mathrm{L}$, give $20 \mathrm{~mL}$ of orange juice orally.

If symptomatic, administer a $5 \%$ dextrose infusion at $4 \mathrm{~mL} / \mathrm{kg} / \mathrm{hour}$.

- The child should not be offered candies, soft drinks or syrups.

- In case of dehydration, ask the patient's physician about oral rehydration solutions (ORS) intake.

- Medications may be given in tablets, but DO NOT ADMINISTER SYRUP.

- Maximum daily glucose for this patient:

If you have any questions, call:

E-mail: @. 